\title{
Health Reform in Minnesota: An Analysis of Complementary Initiatives Implementing Electronic Health Record Technology and Care Coordination
}

\author{
Karen Soderberg ${ }^{1 *}$, Sripriya Rajamani ${ }^{2}$, Douglas Wholey ${ }^{3}$, Martin LaVenture ${ }^{4}$
}

1. Office of Health Information Technology, Minnesota Department of Health, St. Paul, Minnesota

2. Public Health Informatics Program, School of Public Health, University of Minnesota, Minneapolis, Minnesota

3. Public Health Informatics Program, School of Public Health, University of Minnesota, Minneapolis, Minnesota

4. Office of Health Information Technology, Minnesota Department of Health, St. Paul, Minnesota

\section{Abstract}

Background: Minnesota enacted legislation in 2007 that requires all health care providers in the state to implement an interoperable electronic health record (EHR) system by $2015.100 \%$ of hospitals and $98 \%$ of clinics had adopted EHR systems by end of 2015. Minnesota's 2008 health reform included a health care home (HCH) program, Minnesota's patient centered medical home. By end of 2014, $43 \%$ of $\mathrm{HCH}$ eligible clinics were certified with 335 certified $\mathrm{HCHs}$ and $\mathbf{4 3 0}$ eligible but not certified clinics.

Objectives: To study the association between adoption and use of EHRs in primary care clinics and $\mathrm{HCH}$ certification, including use of clinical decision support tools, patient registries, electronic exchange of patient information, and availability of patient portals.

Methods: Study utilized data from the 2015 Minnesota Health Information Technology Clinic Survey conducted annually by the Minnesota Department of Health. The response rate was $80 \%$ with 1,181 of 1,473 Minnesota clinics, including $662 \mathrm{HCH}$ eligible primary care clinics. The comparative analysis focused on certified HCHs (311) and eligible but not certified clinics (351).

Results: $\mathrm{HCH}$ clinics utilized the various tools of EHR technology at a higher rate than non-HCH clinics. This greater utilization was noted across a range of functionalities: clinical decision support, patient disease registries, EHR to support quality improvement, electronic exchange of summary care records and availability of patient portals. HCH certification was significant for clinical decision support tools, registries and quality improvement.

Conclusions: $\mathrm{HCH}$ requirements of care management, care coordination and quality improvement can be better supported with EHR technology, which underscores the higher rate of utilization of EHR tools by HCH clinics. Optimizing electronic exchange of health information remains a challenge for all clinics, including $\mathrm{HCH}$ certified clinics. This research presents the synergy between complementary initiatives supporting EHR adoption and $\mathrm{HCH}$ certification. Ultimately, improvement in health outcomes depends on effective intersection of people, processes and technology. 
Keywords: Informatics; electronic health records; health care homes; health care reform; patient care management

Correspondence: Karen.Soderberg@state.mn.us

DOI: 10.5210/ojphi.v8i3.7094

Copyright @2016 the author(s)

This is an Open Access article. Authors own copyright of their articles appearing in the Online Journal of Public Health Informatics. Readers may copy articles without permission of the copyright owner(s), as long as the author and OJPHI are acknowledged in the copy and the copy is used for educational, not-for-profit purposes.

\section{Introduction}

Minnesota has a strong health reform environment supported by policies and programs related to e-Health and Patient Centered Medical Home (PCMH). Policy makers in Minnesota recognized that more effective use of EHRs, including timely exchange of information is needed to improve quality and safety of care, help control costs, and improve population health. In 2007, Minnesota enacted legislation that requires all health care providers in the state to implement an interoperable EHR system by January 1, 2015 (Minn. Stat. §62J.495) [1]. Minnesota’s EHR mandate predates the Centers for Medicare and Medicaid Service's (CMS) EHR incentive program [2], commonly known as "meaningful use”. The Minnesota EHR mandate also differs in that includes a broad range of providers extending across the continuum of health and care. The state-wide program to support implementation of the EHR mandate is led by the Minnesota Department of Health (MDH) with guidance from the Minnesota e-Health Initiative and its eHealth Advisory Committee [3]. By 2015 all of Minnesota’s hospitals and nearly all ambulatory clinics had adopted EHRs (refer Figure 1)[4]. Other settings not eligible for the CMS EHR incentive program have also advanced in adopting EHR systems.

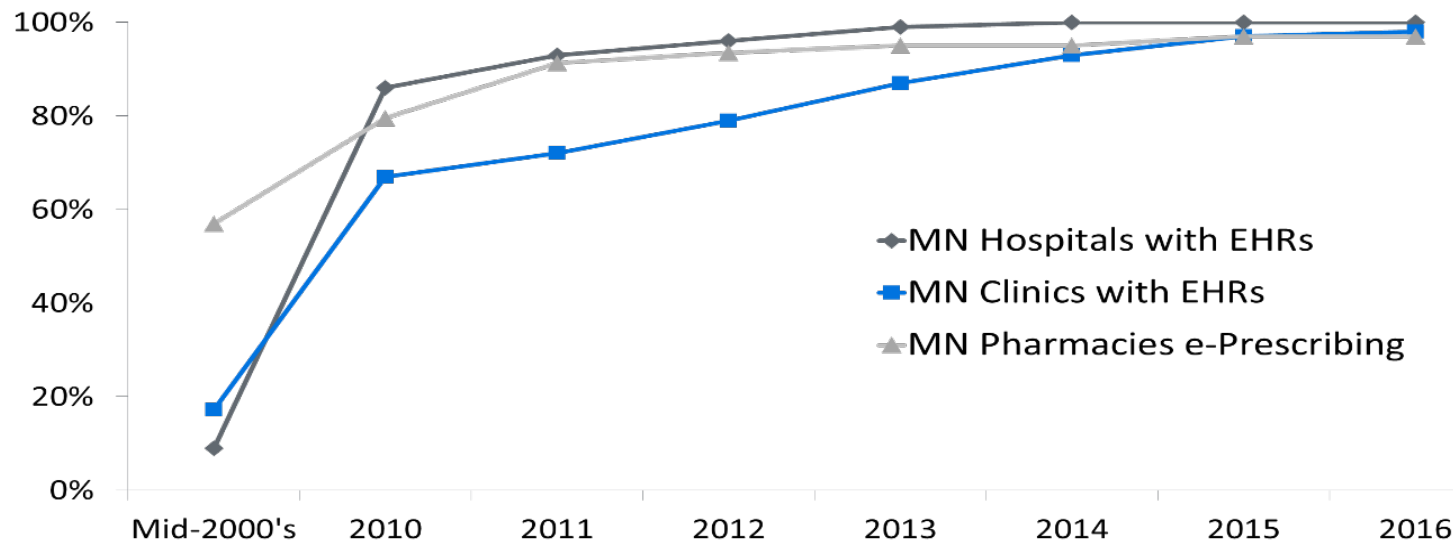

Figure 1: Trends in EHR Adoption in Minnesota

In 2008 the Minnesota Legislature enacted comprehensive health reform legislation comprising of elements targeting population health, market transparency, payment reform and consumer engagement and intended to improve the affordability, access and quality of care [5]. An important element of this legislation is the Health Care Homes $(\mathrm{HCH})$, which is Minnesota's 
version of PCMH. The Minnesota HCH initiative is a joint effort of MDH and the Minnesota Department of Human Services (DHS) (Minn. Stat. §§256B.0751- 256B.0753) [6]. HCH was implemented as "an approach to primary care in which primary care providers, families, and patients work in partnership to improve health outcomes and quality of life for individuals with chronic health conditions and disabilities."'[7]. HCH comprises of both redesign of care delivery and payment reform. $\mathrm{HCH}$ certification requirements included coordination of care, documenting patient conditions and treatments, establishing registries of participating patients and supporting patient engagement. Certification is voluntary, but only certified HCHs are eligible to receive the care coordination payment. MDH began certifying HCHs in 2010 and "eligible" clinics included any that provide primary care services and are located in Minnesota. By end of 2014, 43\% of $\mathrm{HCH}$ eligible clinics were certified with 335 certified HCHs and 430 eligible but not certified clinics (Figure 2).

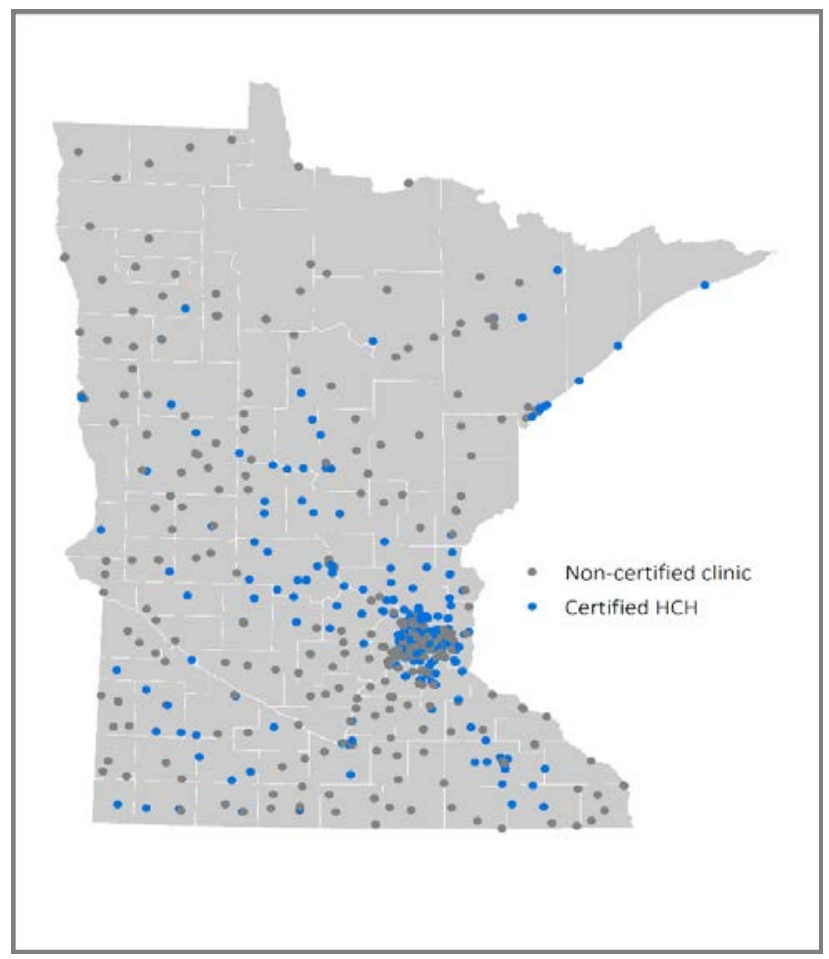

Figure 2: Certified Health Care Homes in Minnesota

Literature review points to the role of health information technology in facilitating the movement towards patient centered medical care [8,9], but it's also been noted that just technology is not adequate and requires needed functionalities (e.g. efficient data exchange and interoperability; notifications on patient status across settings; reporting activities; monitoring patients; better fit with clinical workflow) to impact cost and quality [9-13]. Prior studies examining PCMH and EHR have shown variability in use of tools [14] and have pointed to payment reform as a critical element in influencing care processes rather than EHR alone [15].

This research examines synergies between the two programs on EHRs and HCH by studying the association between adoption and use of EHRs in primary care clinics and HCH certification. In addition, the study presents detailed view at a state level to understand the effect of these programs. Specifically, this analysis considers the utilization of EHR systems among 
Minnesota's HCH eligible clinics, including use of clinical decision support tools, patient registries, electronic exchange of patient information, and availability of patient portals.

\section{Methods}

We used data from the 2015 Minnesota Health Information Technology (HIT) Clinic Survey, a cross-sectional study conducted annually by the Minnesota Department of Health. Clinic(s), for the purpose of this study, means any location where ambulatory clinical care services are provided for a fee by one or more physicians in Minnesota. The 65-question online survey was administered from February 18 to March 17, 2015, and included questions characterizing the adoption and utilization of technology, as well as health information exchange activity. All physician clinics in Minnesota were required to register and complete the survey under the Minnesota Statewide Quality Reporting and Measurement System (SQRMS; Minnesota Rules, Chapter 4654) [16]. The response rate was $80 \%$ with 1,181 of 1,473 Minnesota clinics responding [17].

Eligible primary care clinics are identified through the SQRMS registry and matched to the HIT survey data, resulting in a sub-set of $662 \mathrm{HCH}$ eligible clinics that responded to the survey. The comparative analysis focused on certified HCHs (311) and eligible but not certified clinics (351). EHR capabilities related to HCH requirements of care management and care coordination were characterized as the use of clinical decision support (CDS) tools, utilization of patient disease registries, electronic exchange of summary care records, and availability of patient portals.

The HIT clinic survey is administered at the health system level, meaning that a single response for a health system is attributed to all clinics within that system when all of those clinics have implemented the same EHR and EHR functionalities. Since health systems implement EHRs system-wide, the health system is likely to be an accurate respondent of EHR functionality in its clinics. Because not all of the $\mathrm{HCH}$ eligible clinics within health systems are certified, the comparisons include both certified and not certified clinics within a health system. We compared the difference between certified and not certified clinics using logistic models regressing the presence of a tool (e.g., asthma registry, COPD registry, etc.) on an indicator for the type of tool and certification status. The possibility of correlated errors due to clinics nesting in health systems was addressed by including a health system random effect.

\section{Results}

HCH clinics utilized EHR systems more extensively for the metrics examined in this study: use of clinical decision support functionalities, utilization of patient disease registries, use of EHR data to support quality improvement efforts, electronic exchange of summary care records, and availability of patient portals. The difference between certified and not certified clinics was significant $(\mathrm{p}<.05)$ in all models without correcting for nesting within health systems. When correcting for nesting within health systems, $\mathrm{HCH}$ certification was significant at the .05 level for clinical decision support tools, registries, and quality improvement. Differences in patient portals and electronic exchange of patient information were associated with health system differences. 


\section{Utilization of EHR Clinical Decision Support Functions}

Clinical decision support (CDS) functions offered by EHRs, such as automated alerts, guidelines, care plans and reminders, support the care planning and coordination activities required of certified HCHs. All HCH eligible clinics had near universal use of medication guides/alerts. Certified HCHs were stronger utilizers of six CDS tools compared to not certified clinics for: medication guides/alerts (100\% compared to $95 \%)$; preventive care service reminders (98\% compared to $84 \%$ ); patient- or condition-specific reminders (97\% compared to $82 \%$ ); automated reminders for missing labs and tests (95\% compared to 78\%), clinical guidelines based on patient characteristics (95\% compared to 83\%); and chronic disease care plans and flow sheets (86\% compared to $72 \%$ ) (Figure 3).

\section{Patient Registries}

HCHs are required to manage electronic searchable patient registries and related tools to support care coordination. While EHRs are not the only mechanism that can be used for registries, they are an efficient registry tool and certified HCHs extensively used this functionality. More than nine in ten certified HCHs used electronic registries for patients with asthma (98\%), diabetes (96\%), depression (95\%), hypertension (91\%) and vascular disease (90\%). These clinics outperformed not certified clinics by an average of 21 percentage points. Certified HCHs also outperform not certified clinics in maintaining registries for obesity (77\% compared to $44 \%$ ), congestive heart failure (70\% compared to 38\%) and chronic obstructive pulmonary disease (60\% compared to 29\%) (Figure 4).

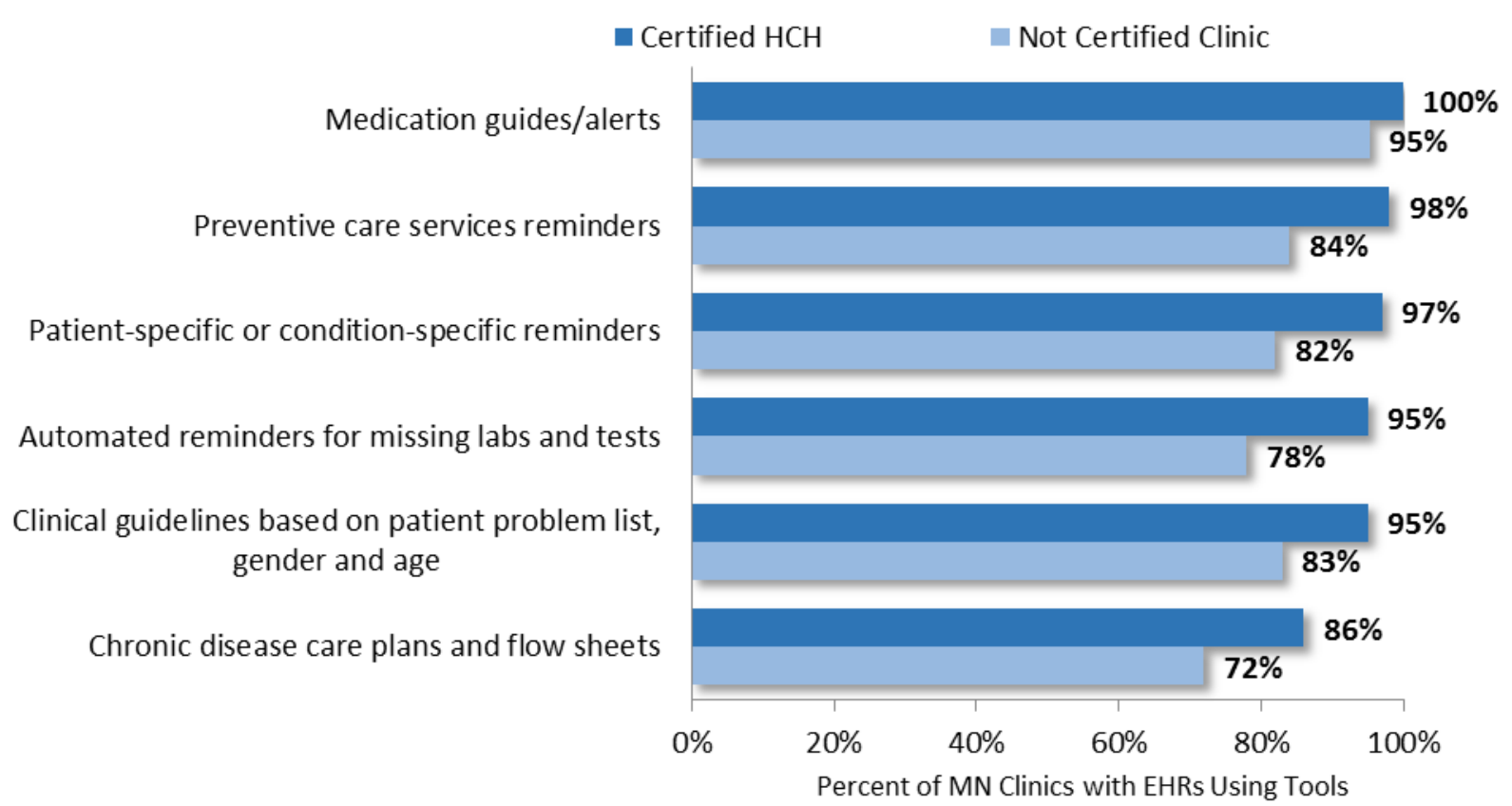

Figure 3: Use of Electronic Clinical Decision Support Tools among HCH Eligible Clinics in Minnesota 


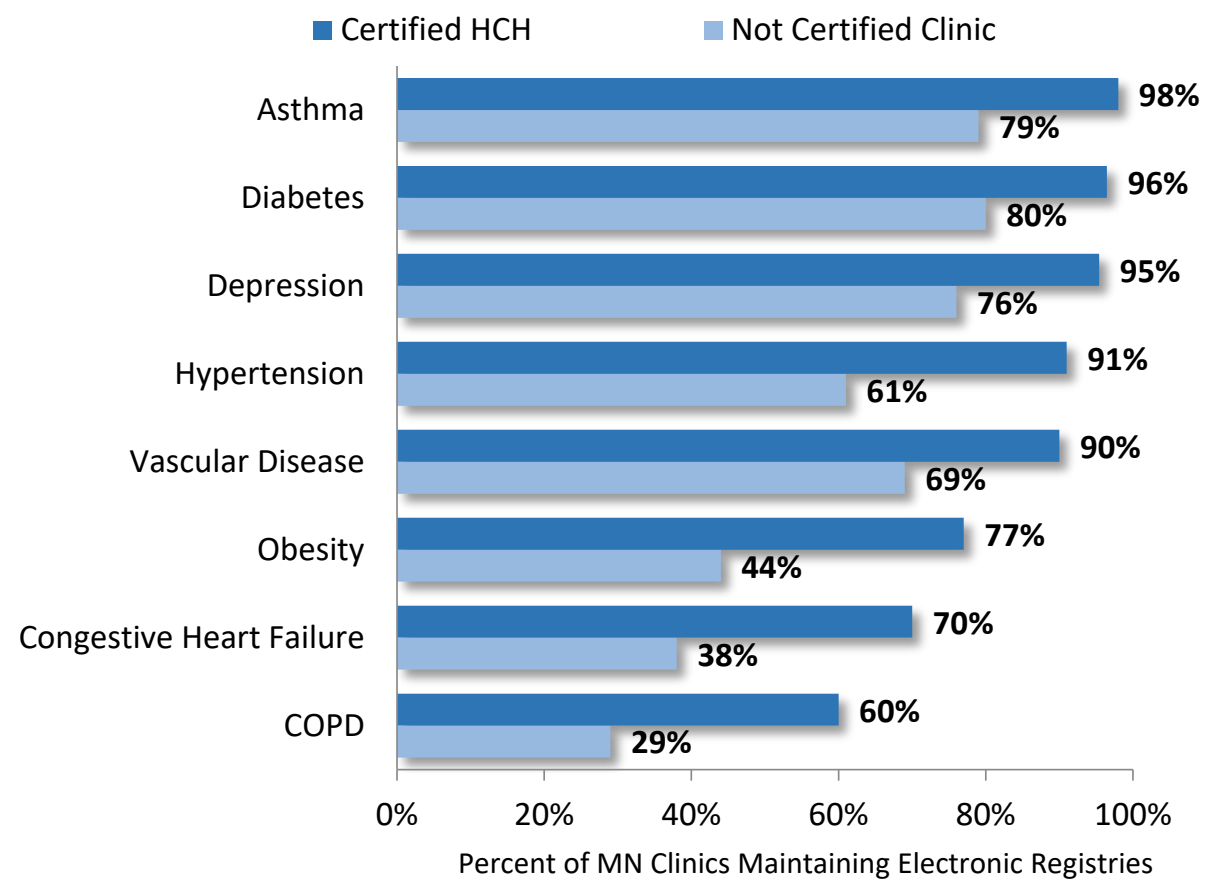

Figure 4: Use of Electronic Disease Registries among HCH Eligible Clinics in Minnesota

\section{Use of EHR Data for Quality Improvement Efforts}

Quality improvement efforts relating to care coordination are important aspects of HCHs as learning organizations, and EHRs offer information tools to support these efforts. Most $\mathrm{HCH}$ eligible clinics utilized data from the EHR for such efforts. Certified HCHs were stronger utilizers of quality improvement activities using EHR data (Figure 5): sharing data with providers (100\% compared to 91\%); creating benchmarks or develop clinical priorities (99\% compared to 86\%); setting goals around clinical guidelines (99\% compared to 83\%); and supporting professional development activities (71\% compared to 50\%).

\section{Electronic Exchange of Patient Information}

A summary of care record is a standardized machine-readable data packet that includes patient information relevant to care providers, such as procedures, diagnoses, problem lists, medication lists, vital signs, and more. Certified HCHs used electronic summary care records more than not certified clinics, with $43 \%$ of certified HCHs using these for $50 \%$ or more of patients who required transition of care, compared to $23 \%$ of not certified clinics (Figure 6).

\section{Availability of Patient Portals}

A patient portal is an internet application maintained by the clinic that allows patients to access their electronic health records and permit two-way communication between patients and their health care providers. Many portals also offer health information for patients to view, such as test results, medication lists, and visit summaries. Ninety-nine percent of certified HCHs offered a patient portal, compared to $91 \%$ of not certified clinics. 


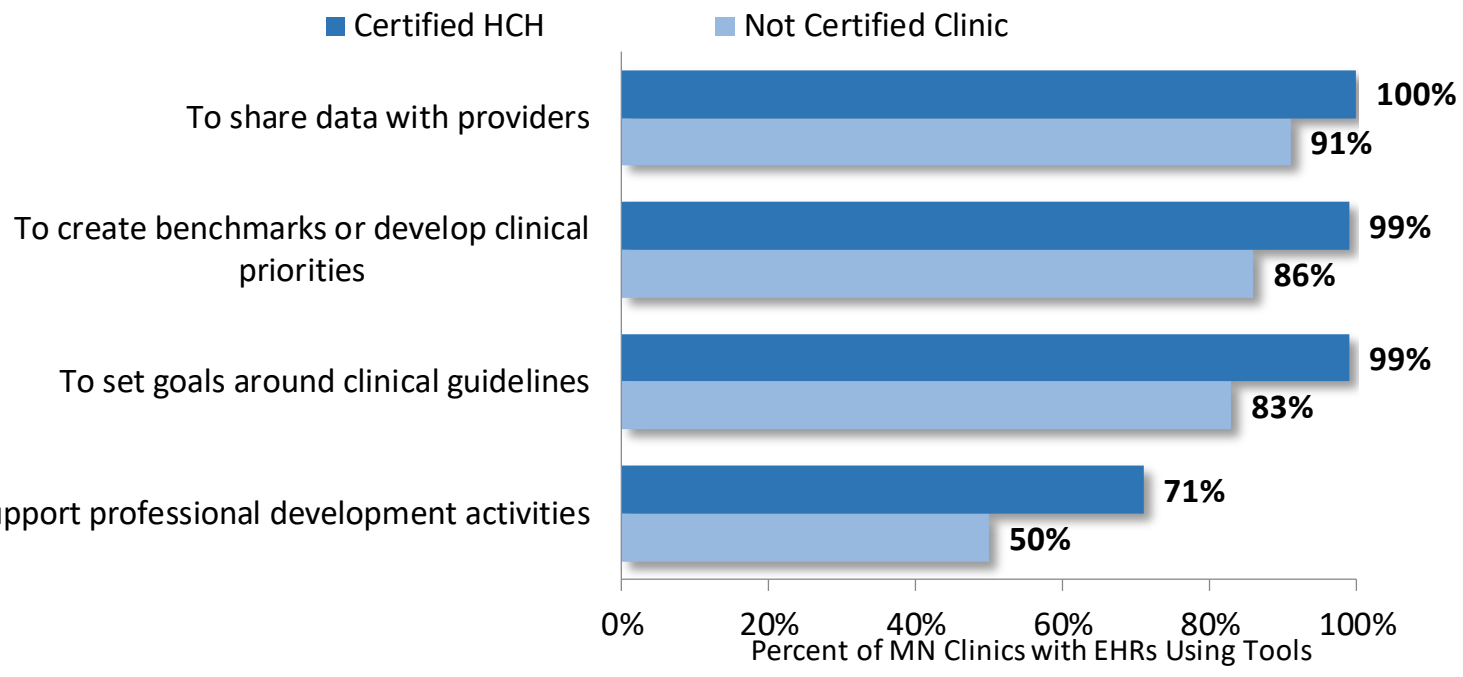

Figure 5: Use of Data from the EHR for Internal Quality Improvement Efforts among HCH Eligible Clinics in Minnesota

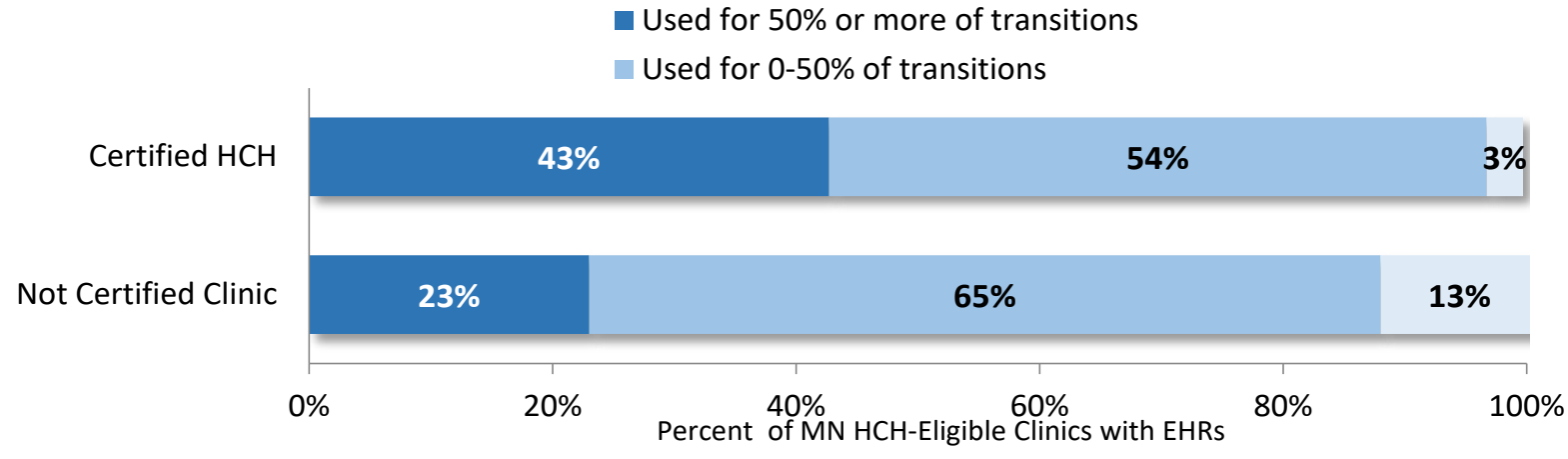

Figure 6: Use of Electronic Summary of Care Record Transitions of Care among HCH Eligible Clinics in Minnesota

\section{Discussion}

While all primary care clinics in Minnesota have high EHR adoption rates, certified HCHs are using the tools that EHR systems offer at a higher rate than eligible but not certified clinics. Though Minnesota's HCH program does not require EHRs, almost all certified clinics had adopted and used this technology extensively, which could be attributed to the EHR mandate in Minnesota. These EHR systems support care management, care coordination, and quality improvement efforts that are needed for Minnesota's health care homes to better manage their patients that have chronic health conditions and disabilities.

Certified HCHs have more extensively implemented clinical decision support tools that can result in improved care, improved patient safety, and lower costs. Certified HCHs also better utilized electronic patient registries, allowing them to better track patients with chronic conditions in order to improve care, observe outcomes, and monitor progress toward care plan goals. Technology offers the opportunity for patients to engage in their health. For patients with 
chronic conditions and/or disabilities, access to their health information, such as through a patient portal, can support patient-centered care by providers and caregivers. Patient portals are offered by majority of $\mathrm{HCH}$ certified clinics.

Our results differ from other studies of EHR implementation in Patient Centered Medical Homes, which found more variable adoption of care coordination tools than our study found [14]. A potential source of this difference is the convergence of complementary health reform initiatives in Minnesota, and has provided opportunity to show distinct differences in the utilization of EHRs among clinics that have committed to EHR adoption and $\mathrm{HCH}$ certification.

The differences between certified and not certified clinics were significant for clinical decision support, registries, electronic exchange of health information, quality improvement, and patient portals when not adjusting for the nesting of clinics in health systems. The differences for clinical decision support registries, and quality improvement were significant when adjusting for nesting in health systems. This suggests that the differences between certified and not certified clinics in electronic exchange of health information and patient portals cannot be separated from health systems efforts to implement both EHRs and HCHs. While the reason for the association between HCHs and EHR implementation in Minnesota may differ, the fact remains that certified HCHs have utilized EHR-based tools extensively.

HCH certification in Minnesota comprise of five standards: access/communication; patient tracking and registry functions; care coordination; care plans and performance reporting and quality improvement[7]. Utilization of EHRs as a tool serves as supporting factor and enhances the meeting of these standards. Ultimately, improvement in health outcomes depends on effective intersection of people, processes and technology. Another factor which may explain the increased use of EHR tools by HCH certified clinics is the maturation of clinics and practices over time. These underscore the need to convey messages that effective utilization of EHRs is critical and not just adoption of the technology.

Electronic exchange of health information is an essential tool to support coordination of care across varied providers, such as primary care, behavioral health, home care, and social support services. All eligible HCHs in Minnesota struggle to electronically exchange summary of care records to support coordination, but certified HCHs outperformed the not certified clinics.

Limitations: As with all surveys, the Minnesota HIT clinic survey is subject to observation error. The survey was completed by clinic administrators/managers with knowledge of EHR implementation at their clinic/health system. Lack of understanding of some terms associated with HIT may led to misinterpretation of survey questions. Furthermore, the respondent may not necessarily have thorough understanding of all items queried in the survey. Another limitation is the possibility of correlated errors due to clinics nesting in health systems; this was addressed by including a health system random effect, as described in the methods section. This work does not examine the factors that influence a clinic to seek HCH certification and its impact on EHR utilization. The study points to use of various EHR functionalities, but additional research is needed to understand if survey responses adequately capture the level of use in a clinic and if the EHR tools are appropriately used. Future directions for this body of research should focus on impact of effective EHR utilization and HCH certification on the quality of care. 


\section{Conclusions}

EHRs and other HIT offer promises to advance individual and population health by providing tools and the right information for providers when they need it to support improved health and clinical care. The real value from investing in and implementing an EHR system comes from using it to support efficient workflows and effective health and clinical decisions. The rapid uptake in EHR technology across the nation, combined with health reform efforts that focus on accountability and care coordination, pose challenges and opportunities for clinical care providers. Opportunities are availability of tools that support decision making, quality improvement and reporting. Challenges remain for clinics in Minnesota to optimize health information exchange. This research highlights the higher utilization of various EHR tools in settings influenced by state policy ( $\mathrm{HCH}$ certification) and has implications for policies and programs. The need to meet various care coordination requirements were likely drivers for better EHR utilization by HCH clinics. These findings suggest that broader health policy objectives can complement overall health reform initiatives. Furthermore, sets of policies can benefit from each other, providing a synergy that each policy alone may not accomplish.

\section{Acknowledgements}

This research was supported by the Health Care Home Evaluation contract done in collaboration with the Minnesota Department of Health and the Minnesota Department of Human Services. The authors would like to thank the Health Care Certification (HCH) program at the Minnesota Department of Health for sharing contextual knowledge and relevant data.

\section{References}

1. Minnesota Statutes. 62J.495 Electronic Health Record Technology. 2007. Available at: https://www.revisor.mn.gov/statutes/?id=62J.495. Accessed November 1, 2016.

2. Centers for Medicare and Medicaid Services (CMS). EHR Incentive Programs. 2009. Available at: www.cms.gov/ehrincentiveprograms. Accessed October 16, 2016.

3. Minnesota eHealth Initiative. Minnesota Interoperable Electronic Health Record Mandate. 2007. Available at: http://www.health.state.mn.us/e-health/hitimp/index.html. Accessed October 19, 2016.

4. Minnesota Department of Health - Office of Health Information Technology. Minnesota eHealth Brief. 2016. Available at: http://www.health.state.mn.us/ehealth/ehealthdocs/briefehealth.pdf Accessed November 2, 2016.

5. Minnesota Department of Health. Minnesota's Health Reform Law. 2008. Available at: http://www.health.state.mn.us/healthreform/about/. Accessed October 29, 2016.

6. Minnesota Statutes. 256B.0751 Health Care Homes. 2008. Available at: https://www.revisor.mn.gov/statutes/?id=256B.0751. Accessed November 1, 2016. 
7. Minnesota Department of Health. Health Care Homes. 2008. Available at: http://www.health.state.mn.us/healthreform/homes/index.html. Accessed October 16, 2016.

8. Health Information Technology. Turning the Patient-Centered Medical Home from Concept to Reality. By David Meyers, MD, Matt Quinn, MBA, and Carolyn M Clancy, MD April 2010 Agency for Healthcare Research and Quality, Rockville, MD https://archiveahrqgov/news/newsroom/commentaries/pcmh-concept-to-realityhtml.

9. Adler-Milstein J, Cohen GR. Implementing the IT infrastructure for health reform: adoption of health IT among patient-centered medical home practices. AMIA Annual Symposium proceedings AMIA Symposium. 2013;2013:11-6. Epub 2014/02/20.

10. Leventhal T, Taliaferro JP, Wong K, Hughes C, Mun S. The patient-centered medical home and health information technology. Telemedicine journal and e-health: the official journal of the American Telemedicine Association. 2012;18(2):145-9. Epub 2012/02/07.

11. Adler-Milstein J, Cohen GR, Markovitz A, Paustian M. The Impact of HIT on Cost and Quality in Patient-Centered Medical Home Practices. AMIA Annual Symposium proceedings AMIA Symposium. 2014;2014:232-9. Epub 2014/01/01.

12. Schapira MM, Sprague BL, Klabunde CN, Tosteson AN, Bitton A, et al. 2016. Inadequate Systems to Support Breast and Cervical Cancer Screening in Primary Care Practice. J Gen Intern Med. 31(10), 1148-55. Epub 06 2016. PubMed http://dx.doi.org/10.1007/s11606-016$\underline{3726-y}$

13. Richardson JE, Vest JR, Green CM, Kern LM, Kaushal R, et al. 2015. A needs assessment of health information technology for improving care coordination in three leading patientcentered medical homes. $J$ Am Med Inform Assoc. 22(4), 815-20. PubMed http://dx.doi.org/10.1093/jamia/ocu039

14. Morton S, Shih SC, Winther CH, Tinoco A, Kessler RS, et al. 2015. Health IT-Enabled Care Coordination: A National Survey of Patient-Centered Medical Home Clinicians. Ann Fam Med. 13(3), 250-56. Epub 05 2015. PubMed http://dx.doi.org/10.1370/afm.1797

15. King J, Patel V, Jamoom E, DesRoches C. 2016. The role of health IT and delivery system reform in facilitating advanced care delivery. Am J Manag Care. 22(4), 258-65. Epub 05 2016. PubMed

16. Minnesota Department of Health. Statewide Quality Reporting and Measurement System. 2008. Available at: http://www.health.state.mn.us/healthreform/measurement/index.html. Accessed October 31, 2016.

17. Minnesota Department of Health - Office of Health Information Technology. Minnesota eHealth Report -Clinics: Adoption and use of EHRs and Exchange of Health Information, 2015. 2015. Available at: http://www.health.state.mn.us/ehealth/summaries/reportclinic2015.pdf. Accessed November 2, 2016. 